\title{
ON FINITENESS CONDITIONS FOR A CONVEX BODY
}

\section{HARVEY COHN}

1. Introduction. In certain problems of geometric number theory, such as the study of critical lattices, it is important to know when a finite convex body surrounding the origin will contain no lattice point other than the origin in its interior. In this brief note we shall discuss a situation in which it suffices to know that only a finite set of lattice points are not interior to the body. In the companion article $[1]^{1}$ we shall show that the results of the present article enable us to reduce the determination of critical lattices in $d$ dimensions to a finite number of steps.

2. Main theorem. Let $K$ be a convex body in d-dimensional euclidean space containing in its interior the hypersphere $\Sigma_{d}(r)$ of radius $r$ and center at the origin $(r<1)$. Let us always consider the lattice of points with integral coordinates. Then if $K$ fails to contain in its interior those lattice points (other than the origin) in a second sphere $\Sigma_{d}\left(C_{d} / r^{d-1}\right)$, then it will contain in its interior no lattice points at all excopt the origin. In fact $K$ will then lie entirely within the second sphere.

Proof. We shall establish the value $C_{d}=2^{d-1} d \Gamma((d+1) / 2) \pi^{-(d-1) / 2}$.

We let $\left(x_{i}\right)$ be an arbitrary point outside of $\Sigma_{d}(r)$, that is, we let $\Sigma x_{i}^{2}=R^{2}>r^{2}$, and we join this point to its negative by a line, namely $\Lambda\left(x_{i}\right)$, through the origin. We let $\Pi\left(x_{i}\right)$ be the $(d-1)$-dimensional hyperplane through the origin and perpendicular to this line, and we let $\Sigma_{d-1}\left(r ; x_{i}\right)$ be the intersection of this plane with the solid sphere $\Sigma_{d}(r)$. For our immediate purposes we define $\kappa\left(r ; x_{i}\right)$ as the (solid) cone formed by the segments joining each point of $\Sigma_{d-1}\left(r ; x_{i}\right)$ with $\left(x_{i}\right)$; and for later purposes we define $\mathrm{K}\left(r ; x_{i}\right)$ as the (solid) cone with spherical base formed by joining with $\left(x_{i}\right)$ each point of $\Sigma_{d}(r)$. Now $\mathrm{K}\left(r ; x_{i}\right)$ will prove useful when we seek the best $C_{d}$, since this solid is a minimal body which any convex body $K$ must contain in order to contain both $\Sigma_{d}(r)$ and $\left(x_{i}\right)$. For our immediate purposes, we need only note that the convex body $K$ will (easily) contain $\kappa\left(r ; x_{i}\right)$ and that the latter body has the very handy value $\omega_{d-1} r^{d-1} R / d$ for its volume, where $\omega_{k}=\pi^{k / 2} / \Gamma(1+k / 2)$, the $k$-dimensional volume of the unit sphere in $k$ dimensions.

Presented to the Society, December 29, 1949; received by the editors March 13, 1950 and, in revised form, August 30, 1950.

${ }^{1}$ Numbers in brackets are items or items and page numbers cited in the bibliography at the end. 
We now note that $\kappa\left(r ; x_{i}\right)$ will contain a lattice point (not the origin) if $R \geqq C_{d} / r^{d-1}$. This is a simple consequence of Minkowski's theorem on convex bodies [3, p. 60]. For the body formed by the union of $\kappa\left(r ; x_{i}\right)$ and $\kappa\left(r ;-x_{i}\right)$ is symmetric and has volume not less than $2^{d}$. Hence there would have to be a lattice point (not the origin) in each of the two symmetric halves. From this the theorem follows easily.

3. Best constant. The preceding estimates can be improved slightly. For instance we may take into account the fact that the convex bodies, $\kappa\left(r ; x_{i}\right)$ and its integral translates, can not pack space more densely than do the $(d-1)$-dimensional spheres (which are precisely their intersections with planes parallel to $\left.\Pi\left(x_{i}\right)\right)$. Then we immediately obtain an improvement of $C_{d}$ to $C_{d}^{\prime}=C_{d} \omega_{d-1} \gamma_{d-1}^{(d-1) / 2} 2^{-(d-1)}$ (where $\gamma_{d}^{1 / 2}$ is the maximum euclidean distance separating the closest points of any $d$-dimensional lattice of determinant unity). But using even the lower estimates [2, pp. 23-24] on $\gamma_{d-1}$, we find the improvement is not substantial, that is, as $d \rightarrow \infty, \log C_{d} \sim \log C_{d}{ }^{\prime}$ $\sim(d / 2) \log d$.

Going in the other direction we can show that there is a best constant for $C_{d}$ as $r \rightarrow 0$. To recapitulate, our main theorem was deduced from Minkowski's theorem. It could also have been deduced from the simultaneous approximation theorem, as the remaining proof will suggest, but the constant $C_{d}$ would not have been as good. The converse of Minkowski's theorem, or the Minkowski-Hwlaka theorem [4], is inapplicable since the problem is not affine invariant. The converse of the simultaneous approximation theorem, or (essentially) Perron's transferal principle [2, p. 67], is, however, applicable and we shall now look to it for a proof that a best $C_{d}$, which we shall call $C_{d}^{*}$, exists.

For the calculation of the best constant, we take a minimal convex body, which is "evasive" of lattice points, namely $K^{*}$ $=\mathrm{K}\left(r ; \rho \theta_{i} /\left(\Sigma \theta_{i}^{2}\right)^{1 / 2}\right)$, reaching to distance $\rho$ from the origin in the direction $\left(\theta_{1}(=1), \theta_{2}, \cdots, \theta_{d}\right)$. The $\theta_{k}$ are chosen, from the Perron transferal principle, to span (say) a real algebraic field of degree $d$ (for convenience, $0<\theta_{k} \leqq 1$ ). Then a constant $g_{d}$ (called $C_{d}$ in [2]) will exist with the property that "the forms $\left|p_{1} \theta_{k}-p_{k}\right|$, for $2 \leqq k \leqq d$, admit a simultaneous approximation no better than $\left(g_{d} p_{1}^{1 /(d-1)}\right)^{-1}$," that is, for $p_{1}>P_{1}(\epsilon)$, the inequality $\left|p_{1} \theta_{k}-p_{k}\right| \geqq\left(\left(g_{d}+\epsilon\right) p_{1}^{1 /(d-1)}\right)^{-1}$ will hold for some $k$ depending on $p_{1}$ and $\epsilon$, and satisfying $2 \leqq k \leqq d$.

We now let $\rho$ increase from $r$ until it reaches the first value $\rho_{0}$ for which $\mathrm{K}^{*}$ has a lattice point $\left(p_{i}\right)$ on its surface. If we took $r$ small enough $\left(<r_{0}(\epsilon)\right)$ then this lattice point would satisfy the inequality 
$p_{1}>P_{1}(\epsilon)$ by the irrationality of the $\theta_{k}$ when $k>1$. If we let $\delta$ $=\left[\Sigma_{i>j}\left(p_{i} \theta_{j}-p_{j} \theta_{i}\right)^{2}\right]^{1 / 2} /\left(\Sigma \theta_{i}^{2}\right)^{1 / 2}$ denote the distance from the lattice point $\left(p_{i}\right)$ to the line $\Lambda\left(\theta_{i}\right)$, then easily $r>\delta$ (since $r$ is the maximum distance from a point of $K^{*}$ to $\left.\Lambda\left(\theta_{i}\right)\right)$, and $\delta>\left[p_{1}^{1 /(d-1)}\left(g_{d}+\epsilon\right)\left(\Sigma \theta_{i}^{2}\right)^{1 / 2}\right]^{-1}$. Therefore using the trivial relationships $\rho_{0} \geqq\left(\Sigma p_{i}^{2}\right)^{1 / 2} \geqq p_{1}$ and $\Sigma \theta_{i}^{2}<d$, we find $\rho_{0}>\left[\left(g_{d}+\epsilon\right)^{d-1} d^{(d-1) / 2} r^{d-1}\right]^{-1}$. This gives us a distance that the convex body $\mathrm{K}^{*}$ can attain without containing an interior lattice point other than the origin. Thus it easily follows that $C_{d} \geqq_{d}^{*} \geqq c_{d}$ $=\left(g_{d} d^{1 / 2}\right)^{-(d-1)}$.

Even using very simple methods, we can improve $c_{d}$ somewhat; but still, on the basis of present information on $g_{d}[2$, p. 72], we can show only that as $d \rightarrow \infty, \log C_{d}^{*} /(d \log d)$ lies between $1 / 2$ and -1 . At any rate, the function $C_{d} / r^{d-1}$, appearing in the main theorem, is seen to contain the best possible power of $r$.

\section{BIBLIOGRAPHY}

1. Harvey Cohn, On the finite determination of critical lattices, Proceedings of the American Mathematical Society vol. 2 (1951) pp. 547-549.

2. J. F. Koksma, Diophantische Approximationen, Ergebnisse der Mathematik und ihrer Grenzgebiete, vol. 4, Berlin, 1936.

3. H. Minkowski, Diophantische Approximationen, Leipzig, 1907.

4. C. A. Rogers, Existence theorems in the geometry of numbers, Ann. of Math. vol. 48 (1947) pp. 994-1002.

WAYNE UNIVERSITY 